\title{
Potentiometric and Spectrophotometric Studies on 8-Quinolinol and Its Derivatives. V. Ionization of 8-Qui- nolinol-5-Sulfonic Acid and 7-Iodo-8-Quinolinol-5-Sulfonic Acid in Aqueous Solution
}

\author{
REINO NÄSÄNEN and AARNE EKMAN \\ Laboratory of Physical Chemistry, Institute of Technology, Helsinki, Finland
}

\begin{abstract}
Gor the investigation of the metal compounds of 8-quinolinol-5-sulfonic 1 and 7-iodo-8-quinolinol-5-sulfonic acids the ionization constants of the acids were required. The earlier determinations are, however, few and ionic strength and temperature are not specified. Therefore a reinvestigation was necessary.
\end{abstract}

These acids are dibasic and their ionization can thus be represented formally by

$$
\begin{aligned}
& \mathrm{H}_{2} \mathrm{~A} \rightleftharpoons \mathrm{HA}^{-}+\mathrm{H}^{+} \\
& \mathrm{HA}^{-} \rightleftharpoons \mathrm{A}^{=}+\mathrm{H}^{+}
\end{aligned}
$$

The law of mass action gives

$$
\left(\mathrm{H}^{+}\right)\left[\mathrm{HA}^{-}\right] /\left[\mathrm{H}_{\mathbf{2}} \mathrm{A}\right]=K_{1}^{\prime}
$$

and

$$
\left(\mathrm{H}^{+}\right)\left[\mathrm{A}^{=}\right] /\left[\mathrm{HA}^{-}\right]=K_{2}^{\prime}
$$

where $\left(\mathrm{H}^{+}\right)$is the activity of the hydrogen ion and the symbols in brackets are concentrations. For the determination of $K_{1}^{\prime}$ and $K_{2}^{\prime}$ the familiar method was used in which sodium hydroxide was added in such an amount that the quotients $\left[\mathrm{HA}^{-}\right] /\left[\mathrm{H}_{2} \mathrm{~A}\right]$ or $\left[\mathrm{A}^{-}\right] /\left[\mathrm{HA}^{-}\right]$ were nearly unity. The $\mathrm{pH}$ measured is then approximately the $\mathrm{p} K$ required. In this case we can write with sufficient accuracy

$$
\left[\mathbf{H A}^{-}\right] /\left[\mathbf{H}_{2} \mathbf{A}\right]=\left(c_{\mathbf{B}}+\left[\mathbf{H}^{+}\right]\right) /\left(c-c_{\mathbf{B}}-\left[\mathbf{H}^{+}\right]\right)
$$

and

$$
\left[\mathrm{A}^{=}\right] /\left[\mathrm{HA}^{-}\right]=\left(c_{\mathrm{B}}-c\right) /\left(2 c-c_{\mathrm{B}}\right)
$$


where $c$ and $c_{\mathrm{B}}$ are the stoichiometric concentrations of the acid and sodium hydroxide added. The more exact equations can easily be derived ${ }^{1}$ but are here unnecessary.

The experimental procedure was the same as previously in this series ${ }^{2}$. All reagents used were the best obtainable.

\section{RESULTS}

Table 1 includes the results on the first ionization of the acids investigated. Each value for the ionization constant is a mean value of four measurements. The deviation of two identical measurements was never greater than 0.01 for $\mathrm{p} K^{\prime}$. The relatively poor solubility in water does not admit the use of higher concentrations than about 0.003 moles per litre for 8-quinolinol-5sulfonic acid and 0.005 moles per litre for 7-iodo-8-quinolinol-5-sulfonic acid. The ionic strength is calculated according to the equation $I=1 / 2 \sum z_{\mathrm{i}}{ }^{2} c_{\mathrm{i}}$, where $c_{\mathrm{i}}$ are molarities. In equation (5) the hydrogen ion concentration is of considerable significance, particularly in the case of the iodine derivative. The hydrogen ion concentration was obtained from the $\mathrm{pH}$ value measured

Table 1. Determination of the first ionization constants of 8-quinolinol-5-sulfonic acid and 7-iodo-8-quinolinol-5-sulfonic acid in aqueous solution of potassium chloride at $25^{\circ} \mathrm{C}$.

8-quinolinol-5-sulfonic acid 7-iodo-8-quinolinol-5-sulfonic acid

\begin{tabular}{|c|c|c|c|c|c|c|c|}
\hline$\sqrt{I}$ & $\mathrm{pH}$ & $\mathrm{pf}_{\mathrm{H}^{+}}+$ & $\mathrm{p} \boldsymbol{K}_{1}^{\prime}$ & $\sqrt{I}$ & $\mathrm{pH}$ & $\mathrm{pf}_{\mathbf{H}^{+}}$ & $\mathrm{p} K_{1}^{\prime}$ \\
\hline 0.040 & 4.131 & 0.020 & 4.081 & 0.061 & 2.947 & 0.026 & 2.487 \\
\hline 0.307 & 4.050 & 0.080 & 3.992 & 0.312 & 2.942 & 0.080 & 2.393 \\
\hline 0.748 & 4.085 & 0.070 & 4.023 & 0.748 & 2.967 & 0.070 & 2.461 \\
\hline 1.050 & 4.160 & 0.018 & 4.115 & 1.050 & 2.982 & 0.018 & 2.551 \\
\hline 1.460 & 4.317 & -0.100 & 4.289 & 1.480 & 3.006 & -0.106 & 2.702 \\
\hline
\end{tabular}

using the activity coefficient values of the hydrogen ion according to Näsänen, Lumme and Mukula ${ }^{2}$.

These results can be represented by the Debye-Hückel equation

$$
\mathrm{p} K_{1}^{\prime}=\mathrm{p} K_{1,0}-\frac{0.509 \sqrt{I}}{1+\alpha \sqrt{I}}+B I
$$

For the 8-quinolinol-5-sulfonic acid the method of least squares gave

$$
\mathrm{p} K_{1,0}=4.092 . \quad \alpha=1.44 . \quad B=0.207 . \quad\left(25^{\circ} \mathrm{C}\right) .
$$

The values for 7-iodo-8-quinolinol-5-sulfonic acid, because of its considerable strength, are not sufficiently accurate for the calculation of the parameters in equation (7) according to the method of least squares. Therefore the thermo- 


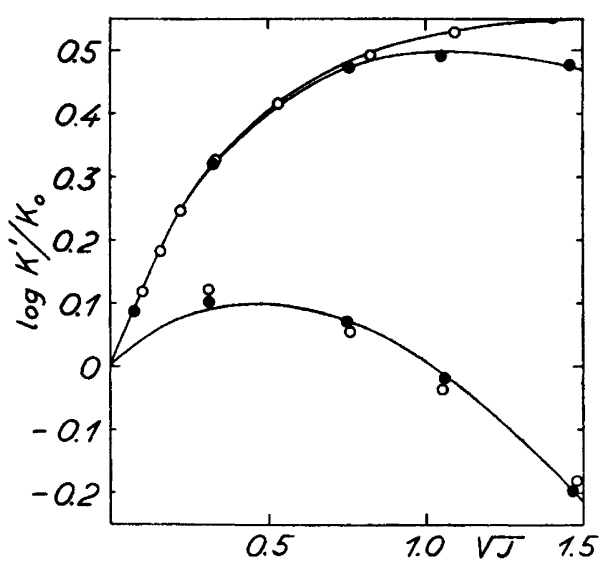

Fig. 1. Ionization constants of 8-quinolinol5-sulfonic acid and 7-iodo-8-quinolinol-5sulfonic acid as functions of ionic strength. First ionization: lower curves. Second ionization: upper curves.

8-quinolinol-5-sulfonic acid.

\% 7-iodo-8-quinolinol-5-sulfonic acid.

dynamic constant was calculated by means of equation (7) using the values of $\alpha$ and $B$ obtained for 8-quinolinol-5-sulfonic acid. For this constant the mean value

$$
\mathrm{p} K_{1,0}=2.514 \pm 0.009 \quad\left(25^{\circ} \mathrm{C}\right)
$$

was obtained. The manner of calculation seems to be justified, for in Fig. 1, where the first ionization constants are represented as functions of ionic strength, we see that the effect of ionic strength is in both cases almost equal within the experimental error.

The results concerning the second ionization are summarized in Table 2. In this case the Debye-Hückel equation

$$
\mathrm{p} K_{2}^{\prime}=\mathrm{p} K_{2,0}-\frac{1.527 \sqrt{I}}{1+\alpha \sqrt{I}}+B I
$$

was used. The method of least squares gave the values of Table 3 .

Table 2. Determination of the second ionization constants of 8-quinolinol-5-sulfonic acid and 7-iodo-8-quinolinol-5-sulfonic acid in potassium chloride solution at $25^{\circ} \mathrm{C}$.

8-quinolinol-5-sulfonic acid

$$
\sqrt{I}
$$

0.0662

0.312

0.744

1.042

1.447

$$
\begin{array}{r}
\mathrm{p} K_{2}^{\prime} \\
8.690 \\
8.455 \\
8.305 \\
8.290 \\
8.300
\end{array}
$$

\begin{tabular}{|c|c|}
\hline$\sqrt{I}$ & $\mathrm{p} K_{2}^{\prime}$ \\
\hline 0.0963 & 7.303 \\
\hline 0.148 & 7.237 \\
\hline 0.216 & 7.172 \\
\hline 0.331 & 7.089 \\
\hline 0.523 & 7.004 \\
\hline 0.815 & 6.928 \\
\hline 1.081 & 6.890 \\
\hline 1.402 & 6.865 \\
\hline
\end{tabular}

$$
\begin{aligned}
& c=3.00 \cdot 10^{-3} \\
& c_{\mathrm{B}}=4.50 \cdot 10^{-3}
\end{aligned}
$$

7-iodo-8-quinolinol-5-sulfonic acid 
Fig. 2. Molar extinction coefficients of the neutral constituents of 8-quinolinol-5-sulfonic acid (middle curve) and 7-iodo-8-quinolinol5-sulfonic acid (upper curve) compared with that of $\mathrm{H}_{\mathbf{2}} \mathrm{Ox}^{+}$(lower curve).

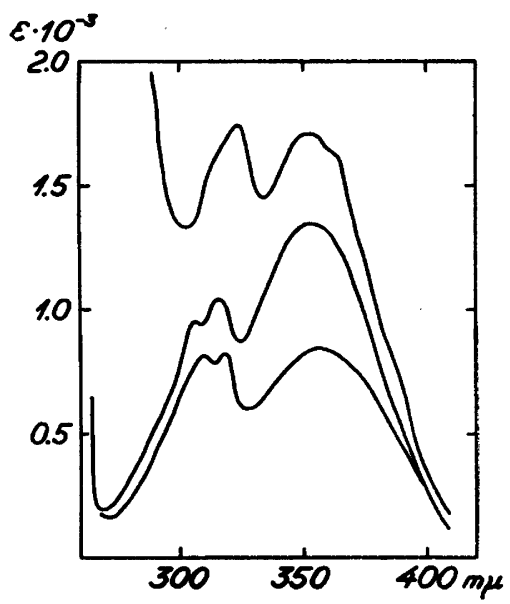

Table 3.

8-quinolinol-5-sulfonic acid

$\mathrm{p} K_{2,0}$

$\alpha$

$B$
8.776

1.62

0.088 7-iodo-8-quinolinol-5-sulfonic acid

7.417

1.71

0.0386

In Fig. 1 the second ionization constants are represented as functions of ionic strength. The effect of temperature on $\mathrm{p} K_{2}^{\prime}$ was preliminary investigated over the range $15^{\circ}-45^{\circ} \mathrm{C}$. For both acids the decrease of $\mathrm{p} K_{2}^{\prime}$ was, on average, 0.095 for one degree rise in temperature.

\section{DISCUSSION}

van Urk ${ }^{3}$ has investigated the indicator properties of 7-iodo-8-quinolinol5-sulfonic acid. Feldman and Powell ${ }^{4}$ have determined colorimetrically the ionization constants of this acid; the ionic strength and temperature are not given. From their values we obtain $\mathrm{p} K_{1}^{\prime}=2.44$ and $\mathrm{p} K_{2}^{\prime}=7.15$. These values are in good agreement with our values at ionic strength $0.05\left(25^{\circ} \mathrm{C}\right)$, for which we obtained $\mathrm{p} K_{1}^{\prime}=2.44$ and $\mathrm{p} K_{2}^{\prime}=7.17$.

Phillips and Merritt ${ }^{5}$ have determined spectrophotometrically the ionization constants of 8-quinolinol-5-sulfonic acid and have obtained $\mathrm{p} K_{1}^{\prime}=4.004$ and $\mathrm{p} K_{2}^{\prime}=8.86$ of which the first constant is in relatively good agreement with our value but the second constant differs somewhat more. Temperature and ionic strength are not stated. No other determinations seem to have been made. 


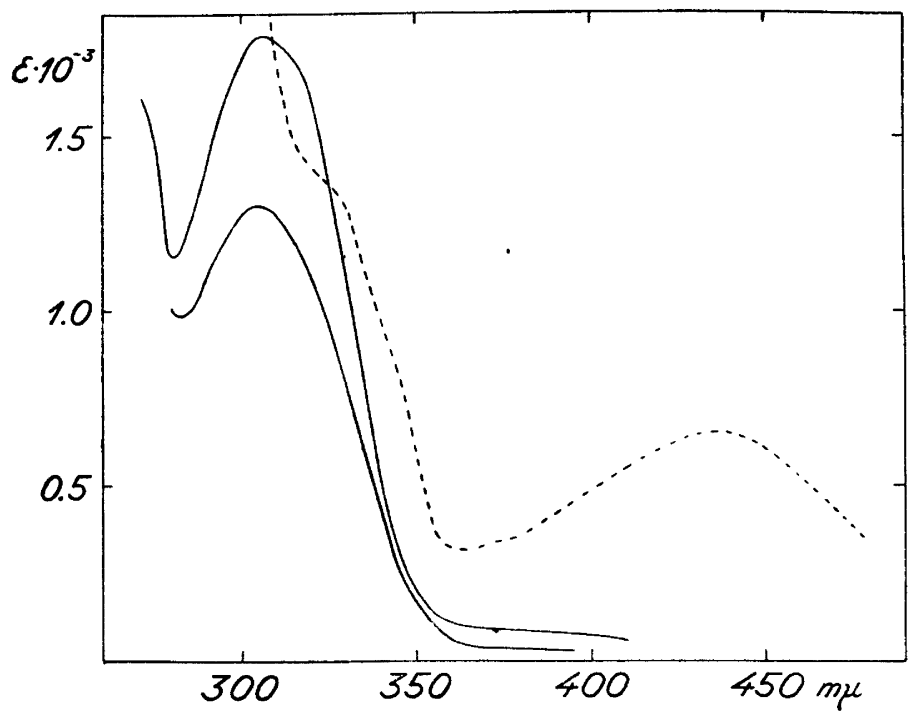

Fig. 3. Molar extinction coefficient of the $\mathrm{HA}^{-}$ions of 8-quinolinol-5-sulfonic acid (upper full line) and 7-iodo-8-quinolinol-5-sulfonic acid (dotted line) compared with that of $\mathrm{HOx}$ (lower full line).

The earlier authors describe these compounds as typical ampholytes. Thus in acidic solution the reagents should exist as positive ions. The titration curve shows, however, that in moderately acidic solutions the reagents exist as neutral molecules. Because of the great acidic strength of the sulfonic group it is very probable that the reagents exist in acidic solutions as zwitterions like

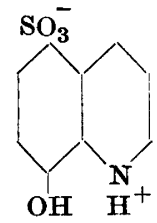

For the same reason the importance of ions like $\mathrm{H}_{3} \mathrm{~A}^{+}$seems to be slight. The effect of ionic strength on ionization also shows that the compounds in question are dibasic acids. The limiting theoretical slope of the Debye-Hückel equation is namely for $\mathrm{p} K_{1}^{\prime}+0.509$ and for $\mathrm{p} K_{2}^{\prime}+1.527$ in the case of the sulfonic acid derivatives but -0.509 and +0.509 respectively for 8-quinolinol ${ }^{2}$.

In figures $2-4$ the ultraviolet spectra of the three constituents $\mathrm{H}_{2} \mathrm{~A}$, $\mathrm{HA}^{-}, \mathrm{A}^{=}$of the acids investigated are compared with the spectra of the corresponding constituents of the parent compound 8-quinolinol: $\mathrm{H}_{2} \mathrm{O}^{+}, \mathrm{HOx}, \mathrm{Ox}^{-}$. 
Fig. 4. Molar extinction coefficients of the $\mathrm{A}^{=}$ions of 8-quinolinol-5-sulfonic acid (upper full line) and 7-iodo-8-quinolinol5-sulfonic acid (dotted line) compared with that of $\mathrm{Ox}^{-}$(lower full line).

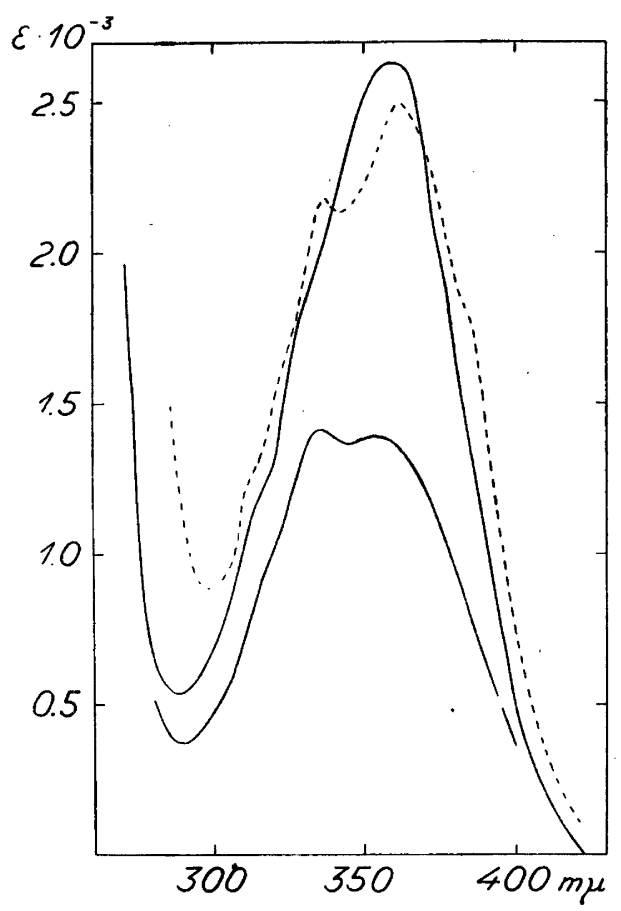

These spectra can be obtained directly. The spectrum of $\mathrm{H}_{2} \mathrm{~A}$ was obtained using a solution with a slight excess of a strong acid and the spectrum of $\mathrm{A}^{=}$ using an excess of strong hydroxide. The spectrum of $\mathrm{HA}^{-}$was obtained using a buffer solution with a $\mathrm{pH}$ corresponding to the first equivalent point. At this $\mathrm{pH}$ the constituents $\mathrm{H}_{2} \mathrm{~A}$ and $\mathrm{A}^{=}$have negligible effect on the spectra. In Fig. 2 the spectra of $\mathrm{H}_{2} \mathrm{~A}$ and $\mathrm{H}_{2} \mathrm{Ox}^{+}$are represented. About the same maxima appear in all cases. The effect of the substituents is, however, distinct. The maximum at $355-360 \mathrm{~m} \mu$ (which can be related to the polar structure II of 8-quinolinol)

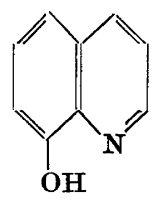

I<smiles></smiles>

II<smiles>O=C(I)c1ncccc2cccc1-2</smiles>

III

has been strengthened in the sulfonic acid derivatives. Also the double maximum of the sulfonic acid derivatives at $305-315 \mathrm{~m} \mu$ is stronger than in the spectrum of $\mathrm{H}_{2} \mathrm{Ox}^{+}$, especially that of the 7-iodo-8-quinolinol-5-sulfonic acid. 
In the latter case a shift to the longer wavelength appears. This maximum at $325 \mathrm{~m} \mu$ also occurs as a little shoulder in the spectrum of the constituent $\mathrm{HA}^{-}$of 7-iodo-8-quinolinol-5-sulfonic acid, as we see in Fig. 3, where the spectra of $\mathrm{HA}^{-}$and $\mathrm{HOx}$ are represented, but is hardly noticeable in the spectrum of $A^{=}$represented in Fig. 4. We further see from Fig. 3 that the constituent $\mathrm{HA}^{-}$of the iodine derivative has a maximum at $435 \mathrm{~m} \mu$. This maximum does not appear in the spectra of the two other constituents of this derivative, probably because of the greater importance of the polar structures in these cases. From Fig. 4 we see that the constituent $A^{=}$of 8-quinolinol-5sulfonic acid has a very strong maximum at $355 \mathrm{~m} \mu$ which corresponds to the structure II, but that the maximum at $335 \mathrm{~m} \mu$ has degenerated to a shoulder. The constituent $\mathrm{A}^{=}$of the iodine derivative still has a relatively strong maximum also at $335 \mathrm{~m} \mu$. The spectra of $\mathrm{A}^{=}$, like $\mathrm{Ox}^{-}$display shoulders at 305-315 $\mathrm{m} \mu$. The spectra thus show that the substituent $\mathrm{SO}_{3}^{-}$increases the importance of the quinoid structure II and the substituent I the importance of the polar structure III as expected. Both substituents raise the acid strength considerably as expected.

\section{SUMMARY}

The ionization of 8-quinolinol-5-sulfonic acid and 7-iodo-8-quinolinol-5sulfonic acid has been investigated. The first and second ionization constants of these dibasic acids, previously treated as typical ampholytes, have been determined. The effect of ionic strength on the ionization has been investigated in potassium chloride solution. The ultraviolet spectra of the three constituents of these acids in aqueous solution have been measured and compared with those of 8-quinolinol. The structures and the effect of substitution on the ionization have been discussed.

This work is part of an investigation, supported by the Finnish Research Council.

\section{REFERENCES}

1. Kilpi, S. Acta Chem. Scand. 6 (1952) 475.

2. Näsänen, R., Lumme, P., and Mukula, A-L. Acta Chem. Scand. 5 (1951) 1199.

3. van Urk, H. W. Z. anal. Chem. 77 (1929) 12.

4. Feldman, H. B., and Powell, A. L. J. Am. Chem. Soc. 62 (1940) 3107.

5. Phillips, J. P., and Merritt, L. L. J. Am. Chem. Soc. 70 (1948) 410. 